\title{
FUNDAMENTAL DETECTION AND ESTIMATION LIMITS IN SPIKE SORTING
}

\author{
Mona A. Sheikh, Don H. Johnson \\ Elec. \& Comp. Engineering Dept. MS-366 \\ Rice University \\ Houston TX 77005-1892
}

\begin{abstract}
Spike sorting refers to the detection and classification of electric potentials (spikes) from multi-neuron recordings, a difficult but essential pre-processing step before neural data can be analyzed for information content. While several spike sorting algorithms have been proposed, our goal is to determine the ultimate limits of spike classification and to characterize this error, regardless of spike sorting algorithm. We account for the major factors influencing the sorting procedure: SNR, relative amplitude ratio and inter-spike correlation in time and waveform morphology. Using an ideal detection/estimation system we calculate detection probabilities and time delay estimation errors as they vary with these parameters, establishing upper bounds on spike classification in terms of these metrics.
\end{abstract}

Index Terms - Spike sorting, detection, estimation

\section{INTRODUCTION}

Neurons in our brain communicate by means of action potentials, sometimes known as spikes. These brief electric pulses, lasting no more than a few milliseconds each, propagate from a neuron to other neurons through axonal branches. Understanding the ins and outs of spiking activity forms the basis for addressing a variety of neuroscience questions about how the brain works. In particular, neuroscientists are interested in "cracking the neural code": deciphering how information is encoded in spike sequences. Spiking activity has recently been used to develop a brain-computer interface for paralyzed individuals [1].

In one recording approach, electrode probes are inserted in the extracellular medium to pick up the electrical activity of neurons. The stream of spike data thus obtained is studied for its temporal and statistical characteristics to formulate conjectures on how neural activity represents information. We define the spike sorting problem to consist of 3 steps in general: (i) detecting the presence of spikes (ii) estimating each spike's time of occurrence, and (iii) classifying the detected spikes according to the neuron that produced each of them.

$\{m s h e i k h, d h j\} @$ rice.edu

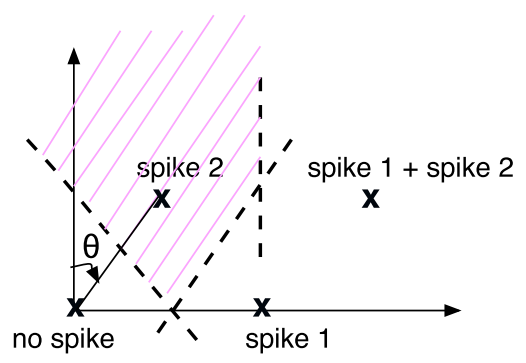

Fig. 1. Spike signal constellation model showing detection region boundaries for spike 2 detection.

Our approach to spike sorting is not to develop a new algorithm. We are interested in characterizing the theoretical limits to which spikes can be correctly identified, regardless of spike sorting algorithm. We model spike sorting as a multiple hypothesis detection problem, and calculate detection probabilities and timing estimation errors from this model using an optimal detector. In this way we quantify the limitations in detecting whether, and if so, when, a spike has occurred.

The major sources of error are (i) additive non-stationary noise (due to background spiking and electronic hum), (ii) unpredictable waveform changes (due to electrode drift and the non-homogeneous conducting medium), (iii) temporal overlaps and (iv) similarity between spike waveforms that hinders classification. Assuming a point source model for a neuron [2], we showed that small-spike interference can be approximated as gaussian [3] for our analysis. These error sources in a neural recording can be parameterized by SNR, spike amplitude ratio and inter-spike correlation.

\section{SPIKE SIGNAL CONSTELLATION MODEL}

The constellation diagram that we propose is different from traditional signal constellation diagrams in that spike correlation takes into account error due to overlap in time in addition to the zero-delay correlation, what we call the morphological (maximal) correlation of the waveform template. As shown in Figure 1, the four constellation points denote the four different hypotheses that describe a two-neuron single- 

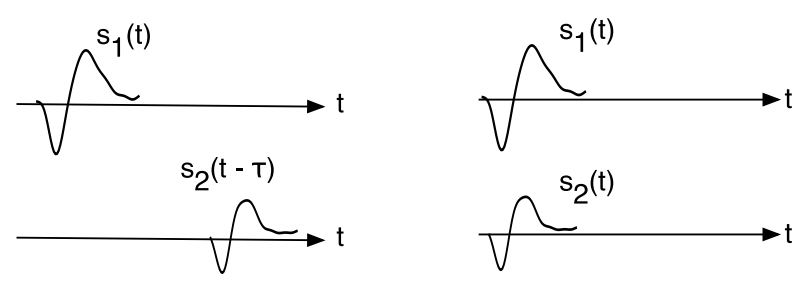

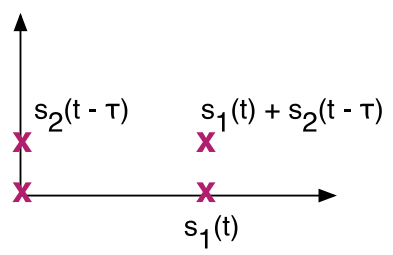

(a)

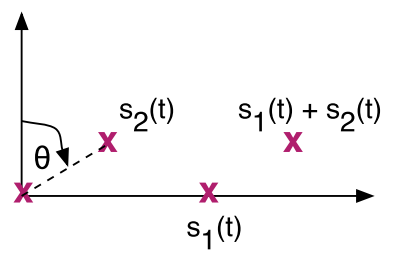

(b)
Fig. 2. Constellation for (a) non-overlapping spikes separated by time delay $\tau$ and (b) completely overlapping spikes (b). Distance of $s_{1}$ and $s_{2}$ from the origin represents the respective amplitudes $A_{1}, A_{2}$ of the two spikes, and their geometric sum corresponds to the constellation point representing both spikes occurring within the observation window.

electrode recording. They form a parallelogram having a slant angle of $\theta$. We take spike 1's waveform as a coordinate, here the $x$-coordinate, so that spike 2 's constellation point is determined by the Gram-Schmidt process. The perpendicular axis to spike 1 represents all spikes orthogonal in time and/or waveform to it. Therefore inter-spike correlation accounts for errors due to both (i) similarity in waveform morphology and (ii) temporal overlap. Any spike that does not overlap in time with spike 1 or is perfectly orthogonal (in the sense of an inner product) to spike 1 has its constellation point located on this perpendicular axis. The angle that spike 2 coordinates make with spike 1 indicates its total correlation with spike 1 . Geometrically, the correlation angle $\theta$ that defines the parallelogram's slant equals $\sin ^{-1} \rho$, where $\rho$ is the inter-spike cross-correlation for a given overlap $\tau$.

$$
\rho(\tau)=\frac{\int_{0}^{T} s_{1}(t) s_{2}(t-\tau) d t}{\sqrt{\int_{0}^{T} s_{1}^{2}(t) d t \cdot \int_{0}^{T} s_{2}^{2}(t-\tau) d t}}
$$

The distances of spike 1 and spike 2's constellation points from the origin indicates their respective amplitudes. The larger the spike amplitude (as determined by SNR), the further from the origin its corresponding constellation point. In our representation, we use the convention that spike 2 has the smaller amplitude $\left(A_{2} \leq A_{1}\right)$. We study not only how SNR affects the amplitude of both spikes simultaneously, but also how variations in spike 2's amplitude with respect to spike 1's affect classification and detection performance.

The ideal detector/estimator system that we envision is a

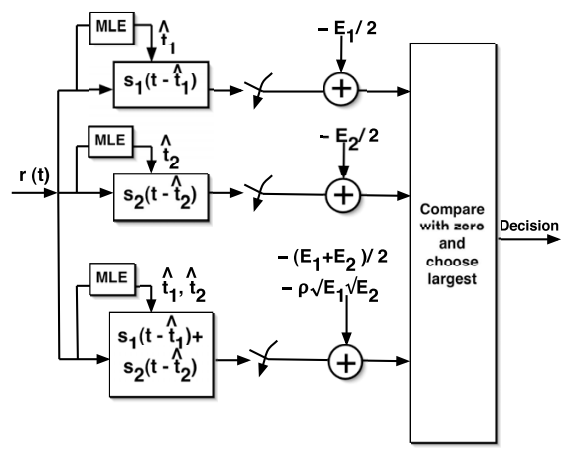

Fig. 3. Matched filter system to calculate upper limits of spike time estimation error and detection probability.

system of 3 matched filters as shown in Figure 3 in which each matched filter knows the template waveform for the spike it is detecting, with the matched filter detecting overlaps testing for all possible combinations of the two spikes. Each of the matched filters must estimate the spike time at which its spike was most likely to have occurred by its ML (Maximum Likelihood) estimate before calculating detection probabilities. As delay is unknown the hypotheses being chosen between are (i) $s_{1}\left(t-\hat{\tau}_{1}\right)$ (ii) $s_{2}\left(t-\hat{\tau}_{2}\right)$ (iii) $s_{1}\left(t-\hat{\tau}_{o v_{1}}\right)+s_{2}\left(t-\hat{\tau}_{o v_{2}}\right)$ (iv) no spike, where $\hat{\tau}_{1}, \hat{\tau}_{2}, \hat{\tau}_{o v_{1}}$ and $\hat{\tau}_{o v_{2}}$ are the times estimated by the matched filter. The observation interval is short enough that the occurrence of two spikes from the same unit is not of concern. A neuron cannot produce a second action potential during its refractory period $(\approx 1 \mathrm{~ms})$ after producing a spike.

\section{LOWER BOUNDS ON SPIKE TIME DELAY ESTIMATION}

We investigate analytical bounds on the estimation error of the times at which spikes occur. We consider the Cramér-Rao lower bound on the mean-squared estimation error for the two-signal delay estimation problem. Assume that two signals have occurred at $\tau_{1}$ and $\tau_{2}$ such that $r(t)=s_{1}\left(t-\tau_{1}\right)+s_{2}\left(t-\tau_{2}\right)+N(t)$. The Fisher information matrix, whose diagonal elements give a lower bound on individual time delay error:

$$
F^{-1}=\frac{N_{0}}{2}\left[\begin{array}{cc}
\frac{1}{1-\dot{\rho}^{2}} \frac{1}{\| \dot{s_{1} \|^{2}}} & \frac{\dot{\rho}^{2}}{1-\dot{\rho}^{2}} \frac{1}{<\dot{s_{1}, \dot{s_{2}}>}} \\
\frac{\dot{\rho}^{2}}{1-\dot{\rho}^{2}} \frac{1}{\left\langle\dot{s_{1}}, \dot{s_{2}}\right\rangle} & \frac{1}{1-\dot{\rho}^{2}} \frac{1}{\| \dot{s_{2} \|^{2}}}
\end{array}\right]
$$

We see that the diagonal elements of $F^{-1}$ depend on $N_{0}$, $\left\|\dot{s_{1}}(t)\right\|$ (which also appears in the Cramér-Rao lower bound for single spike timing error) and $\dot{\rho}$, the correlation coefficient of the signal derivatives. From this bound we see that the larger $\left\|\dot{s}_{1}(t)\right\|$, the lower the mean squared estimation error of $\tau_{1}$, implying that a narrower or higher frequency spike 


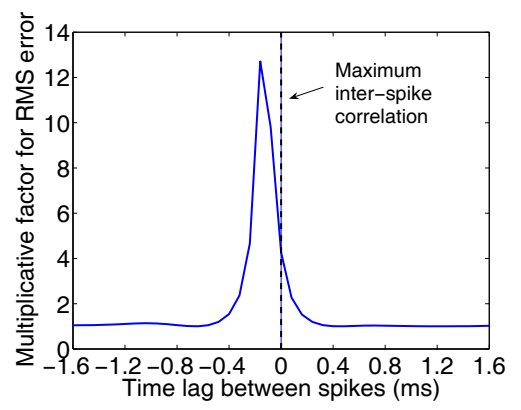

Fig. 4. Variation of $\left(1-\dot{\rho}^{2}\right)^{-1}$ with $\tau$, corresponding to the variation of estimation error between its minimum and maximum value as overlap increases.

has a lower mean-squared error than a broader one, given that $N_{0}$ (SNR) and $\dot{\rho}$ are constant. More interesting is the error dependence on $\dot{\rho}$. From this expression we see that the mean squared error in estimating either $\tau_{1}$ or $\tau_{2}$ increases with $\dot{\rho}$. $\dot{\rho}$ and $\rho$, the correlation coefficient of the signals, are only loosely related, and quantify different waveform similarities. Therefore it is the correlation between the signal derivatives, not the signals themselves, that plays a role in the ultimate time delay estimation error. Figure 4 describes how the RMS error scales with overlap for a given pair of spikes at fixed SNR (implying fixed $N_{0}$ and $\left\|\dot{s}_{1}(t)\right\|$ ). RMS error grows by approximately a factor of 12 from zero overlap. Interestingly we note that the maximum RMS error does not necessarily occur at the point of maximum overlap between the two spikes.

We performed simulations for error estimation using spikes from real neural data with added synthetic noise to compare the error in time estimates with the CRLB for the two-spike case. The resultant variation of estimation error with spike overlap corroborates the CRLB estimation error variation with overlap through $\dot{\rho}$. Figure 5(a) illustrates how the time estimate histograms for spike 2's time vary as the relative position of spike 2 changes with respect to spike 1 , for equal amplitude $s_{1}$ and $s_{2}$. When the spikes do not overlap with each other, the peak for spike 2's time is correctly located and of greater height than the second histogram peak. However, in the central region of the mesh plot when spike 1 and spike 2 overlap, detecting spike 2's location correctly becomes more difficult and the width of the peak increases to a maximum before decreasing again. At close overlaps, the detector may start to locate spike 2 at spike 1's location or at a time between them. These unpredictable variations occur because of the ways in which the two waveforms overlap by either reinforcing or negating each other, changing the interspike correlation value which in turn affects the matched filter's time MLEs. The peak estimate may lie in between the true peaks of the two spikes depending on how the two overlapping spikes reinforce each other. Figure 5(b) shows spike 1 and spike 2 overlapping to reinforce each other with a time lag of $0.33 \mathrm{~ms}$ ( 2 samples) between them. Here the detector most

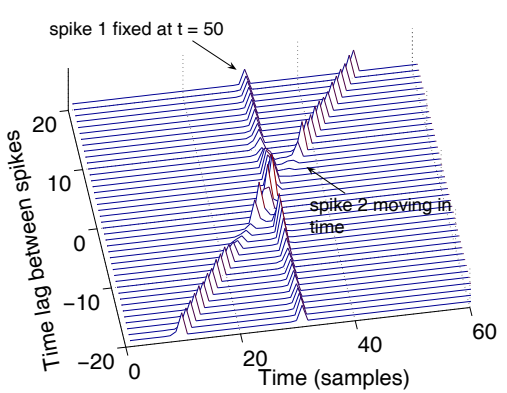

(a)

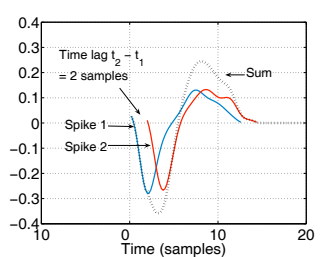

(b)
Fig. 5. (a) Time delay estimates of spike 2 varying with overlap. Spike 1 and spike 2 are equi-amplitude. (b) Two spikes overlap to reinforce each other, creating a new peak between the true spike times.

frequently estimated spike 2 (true $t_{2}=4$ and true $t_{1}=2$ ) to have occurred at $t=3$, when the peak of the overlap signal occurs between the spike times.

Through simulation we calculated the variation of RMS timing error with SNR and relative spike amplitude for twospike delay estimation. We found that the time estimate histograms for either of the two spikes had a bimodal distribution since the detector latches on to anything that looks spike-like. This results in a strong possibility of switching spike times. The heights of the two peaks in the histogram depend on the relative amplitude of the spikes in the recording. The larger a spike is, the taller is the histogram peak at its spike time. Therefore relative spike amplitude plays a large part in determining how probable it is that a spike time will be estimated correctly. Improving either SNR or the relative amplitude of the spike to be detected lowered the RMS timing error, but SNR had a stronger effect on lowering error than spike amplitude ratio. On the other hand, spike amplitude ratio was more influential in improving the height of the histogram peak, corresponding to an improved probability that the time estimate was associated with the correct spike.

\section{DETECTION PROBABILITY}

We investigated detection probabilities after the spike occurrence times have been estimated and found that there is a detection bias towards selecting the overlap hypothesis: regardless of the true hypothesis in the recording, the matched filter system always decided that "both spikes" were present, regardless of SNR. This situation might be expected. Even though Matched Filter III subtracts the amplitude of the overlap template, there is still a large correlation that remains that is compared with the other matched filter outputs. This usually happens with template-based detection and has been noticed experimentally as well [4]. We realize that the Generalized Likelihood Ratio Test (GLRT) is not necessarily optimal 


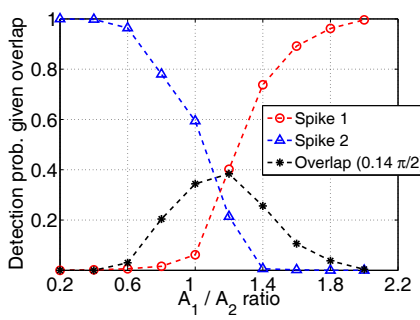

(a)

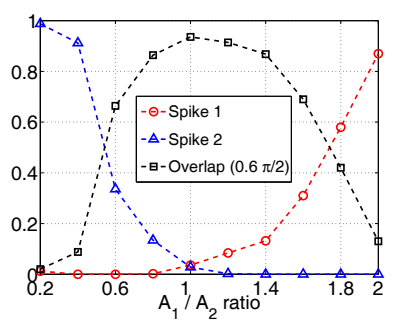

(b)
Fig. 6. Ground truth is that two spikes occur in the recording overlapping with correlation (a) $0.14 \pi / 2$ and (b) $0.6 \pi / 2$. The curves show error probabilities of detecting each of the two spikes individually, and probability of detecting the overlap.

because of the element of uncertainty in parameter estimation as seen in non-coherent detectors [5]. The probability of false alarm and probability of detection are both very high, regardless of SNR. This behavior we see with Matched Filter III; there is always a bias towards saying the spikes are there. Therefore we used an additional threshold to lower the probability of false alarm.

We investigated the variation of detection probability with our parameters of interest. Time delay of the spike is estimated prior to detection. This extra step of delay estimation introduces an additional element of error. Figure 6 illustrates the hit that detection probability takes when delay is unknown for single spike detection. Figure 6(a) and (b) illustrate how detection probability varies with spike amplitude ratio in the case that two overlapping spikes are present in the recording for low and high correlation values. We found that detection probability of the overlap case was best when amplitude ratio was close to 1 . However, depending on how the spikes overlap, detection probability of the overlap may not be as high, or may decrease, especially if they negate each other (here they reinforce each other positively). We notice that the individual probabilities of detecting one of the spikes in the overlap largely depends on the amplitude ratio of the two spikes concerned. The detection probability of the larger spike is always higher and increases with amplitude disparity. The spike shapes are similar enough that the detector latches on to the bigger spike; spike amplitude ratio greatly affects detection probability. However, if correlation is larger (either due to greater temporal or morphological overlap) as in Figure 6(b), we notice that overlap detection probability is better for the same spike amplitude ratio. Moreover, probability of correct overlap detection extends to a wider range of spike amplitude pairs if inter-spike correlation is higher.

\section{CONCLUSIONS}

We have presented a signal constellation model that accounts for three major parameters of influence in the detec- tion/estimation of the spike sorting process: noise (SNR), relative spike amplitude ratio and inter-spike correlation (temporal and morphological waveform overlap). Detection probabilities using an ideal detector can be calculated using this model, or an equivalent matched filter detector/estimator system. Through simulation we found that while relative spike amplitude is important for detection probability, SNR is more crucial for minimizing time delay estimation error. We derived that the increase in RMS error with overlap is governed by maximizing correlation between the signal derivatives, and not the signals themselves. We also showed that overlap detection is best for similar amplitude spikes, but the trade-off for equal amplitude is that RMS error will worsen. Thus spike amplitude plays a large part in correct detection and time estimation if there is more than one spike in the observation interval; even an ideal detector latches on to whichever spike is larger. An important ramification of classification errors during the sorting stage is their effect on interval statistics of the marked point process that the spike train is translated to. The effects of these errors need to be taken into account when analyzing spike trains for information content. We emphasize that the constellation model, detection probability and estimation error results here extend to any detection/estimation problem for spike-like signals that can be parameterized by the same sources of error. In fact, the results that we have presented are applicable to any UWB type communication system in which identification of short pulses and their timing is of concern.

\section{REFERENCES}

[1] D. Santucci, Jerald D. Kralik Mikhail A. Lebedev, and Miguel A. L. Nicolelis, "Frontal and parietal cortical ensembles predict single-trial muscle activity during reaching movements in primates," European Journal of Neuroscience, vol. 22, Issue 6, pp. 1529, 2005.

[2] D.H. Johnson, Array Signal Processing, Prentice Hall, Upper Saddle River, NJ, 1993.

[3] M. Sheikh, Fundamental Limits in Spike Sorting, M.S. Thesis, Rice University, 2006.

[4] G. Wang, Y. Zhou, A. Chen, P. Zhang, and P. Liang, "A robust method for spike sorting with automatic overlap decomposition," Transactions on Biomedical Engineering, vol. 53, pp. 1195-1198, 2006.

[5] P. Moulin, "A detection-theoretic and computational framework for designing geometrically resilient watermarking systems,"

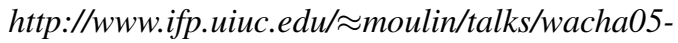
slides.pdf, vol. WaCha, Barcelona, 2005. 